\title{
Risk factors for cardiovascular disease in chronic spinal cord injury patients
}

\author{
H Krum FRACP,${ }^{1}$ L G Howes PhD FRACP,${ }^{1}$ D J Brown FRACP, ${ }^{2}$ G Ungar \\ FACRM,${ }^{2}$ P Moore RN,${ }^{2} \mathrm{~J} \mathrm{~J}$ McNeil PhD FRACP,${ }^{3} \mathrm{~W}$ J Louis MD FRACP ${ }^{1}$ \\ ${ }^{1}$ Clinical Pharmacology Unit, ${ }^{2}$ Spinal Injuries Unit, Austin Hospital, Heidelberg, Victoria \\ 3084, Australia: ${ }^{3}$ Department of Social and Preventive Medicine, Monash University, \\ Prahran, Victoria 3181, Australia.
}

To establish whether the reported increased cardiovascular (CV) morbidity in spinal cord injury (SCI) patients is due to increased levels of established CV risk factors, we assessed overall CV risk in 102 consecutive patients aged 25-64 by calculation of a 'risk factor score' (RFS) derived from the MRFIT study (age, diastolic blood pressure (DBP), total cholesterol (TC) level, cigarettes/day, sex), obtaining a percentile position amongst an age and sex matched peer group from the 1983 Australian Risk Factor Prevalence Study. Chronic SCI patients had a very low overall percentile position of risk of $26.03+15.06$ (mean \pm S.D.) and those patients with SCI for greater than 10 years had only a slightly higher risk position of $33.16 \pm 29.66$. The low relative risk in SCI patients was due mainly to significantly lower DBP levels $(67 \pm 13 \mathrm{~mm} \mathrm{hg})$, as TC levels $(5.38 \pm$ $1.60 \mathrm{mmol} / \mathrm{L}$ ) and cigarette consumption (31\% smokers, mode $11-20 /$ day) were similar to the control population $(83 \pm 12 \mathrm{mmHg}, 5.68 \pm 1.2 \mathrm{mmol} / \mathrm{L}, 28 \%$ smokers, mode $11-20$ /day, respectively).

As other known risk factors such as lipoprotein cholesterol fractions were not included in the RFS index, these were measured in 327 consecutive SCI patients. HDL cholesterol levels, which are negatively correlated with $\mathrm{CV}$ risk, were significantly lower in SCI patients $(1.12 \pm 0.30 \mathrm{mmol} / \mathrm{L})$ compared to controls $(1.35 \pm 0.35 \mathrm{mmol} / \mathrm{L})$ and those patients more than 10 years post SCI had still lower levels $(1.02 \pm 0.40)$.

These data suggest that the reported increased incidence of CV disease in SCI patients is unexplained by increases in BP, TC or smoking. However, low HDL levels may contribute to $\mathrm{CV}$ risk and the role of other risk factors such as increased vascular reactivity remain to be established.

Key words: spinal cord injury; blood pressure; lipoproteins; cigarette smoking; cardiac risk.

\section{Introduction}

The management of patients after spinal cord injury (SCI) has improved dramatically in the last 20 years. Because of this, life expectancy following spinal cord injury (SCI) is now approaching that of the general population and the major causes of morbidity and mortality affecting the general community such as cardiovascular disease ${ }^{1}$ may now be expected to be prevalent in long term SCI patients.

A number of studies have examined the incidence of cardiovascular disease (CVD) in chronic SCI patients. A significantly increased incidence of hypertension and ischaemic heart disease, but not diabetes, was found in SCI patients compared to age and sex matched controls from the Israeli population. $^{2}$ In addition, a Canadian study ${ }^{3}$ noted an increased incidence of death due to heart disease in SCI patients compared to that expected from population studies.

Risk for cardiovascular disease can be assessed from measurement of 'risk factors' in individual patients, the major ones being serum cholesterol levels, blood pressure, 
cigarette smoking, glucose tolerance, physical activity and family history..$^{4,5}$

While risk factors for CVD have not as yet been determined in detail in SCI patients, a number of studies have found low HDL cholesterol levels in this group. ${ }^{6} \mathrm{HDL}$ cholesterol levels have been reported to be inversely related to the incidence of CVD. ${ }^{7}$

To assess the risk of CVD in SCI patients, we investigated the incidence of cardiac risk factors in SCI patients and compared them to those of age and sex matched members of the Australian general population.

\section{Study design}

\section{Subjects}

SCI patients aged 25-64 years attending spinal outpatients for annual routine intravenous pyelogram or isotope renal scan were studied. Cardiac risk factors were measured in 327 consecutive patients, 16\% of whom were female and $84 \%$ male. Twenty nine per cent of all patients had had SCI for greater than 10 years.

In addition, a questionnaire assessment of cardiac risk was performed in the initial 102 of these patients, $19 \%$ of whom were female and $81 \%$ male. Of these patients, $34 \%$ had had their spinal injury for greater than 10 years.

Fully informed consent was obtained from all patients and the protocol was approved by the Austin Hospital Ethical Review Committee.

Retrospective comparisons were made with age and sex matched controls from the 1983 National Heart Foundation Risk Factor Prevalence Study of the Australian community $^{8}$, this study providing the most comprehensive and current data on cardiovascular risk in the Australian population. Whilst a prospective matching of spinal patients with controls would be preferable, the difficulty in selecting an appropriate control group made a prospective study design of this type impossible.

\section{Study procedure}

Measurements were made of body weight, recumbent Dinamap systolic and diastolic blood pressure (after 15 minutes rest), serum cholesterol and lipid fractions, and serum glucose.

The questionnaire assessed cigarette consumption, dietary habits, alcohol consumption, psychological assessment of stress levels, level of physical exercise (5 level semiquantitative assessment with NIL $=$ no exercise to level $4=$ wheelchair athlete) and history of concurrent cardiovascular disease. Male patients were analysed according to age specific percentiles. Because of the low numbers of female patients in the SCI group, all females were analysed as a single group. Patients greater than 10 years post spinal injury were analysed as a separate group.

A cardiac risk factor score was derived from logistic regression coefficients obtained by analysis of data from the Multiple Risk Factor Intervention Trial (MRFIT) ${ }^{4}$ according to the formula: risk score = $0.1091 \times$ age $+0.288 \times$ diastolic blood pressure $+0.008 \times$ total cholesterol $+0.227 \times$ cigarettes per day.

The Australian population was stratified into age and sex specific percentiles from the NHF study ${ }^{9}$ based on the MRFIT risk score and SCI patients were then compared to NHF controls to determine their ageadjusted risk percentile position within the Australian population.

Males and females in the 2 study groups were compared by Student's unpaired t-test unless indicated otherwise.

\section{Results}

\section{Patient demographic data}

Level of spinal injury is shown in Figure 1. Most patients either had cervical or lower thoracic levels of injury. Frankel grade is shown in Figure 2. Most patients were Frankel grade A, ie functionally complete neurological deficit. A lower incidence of hypertension was noted in spinal patients with an increase in diabetes incidence compared to the NHF group (Fig 3). The incidence of ischaemic heart disease, cerebrovascular accident and hypercholesterolemia were similar in the 2 groups. Dietary habits were similar in the 2 groups, the majority of both groups being on no special diet with a slight increase in spinal patients 


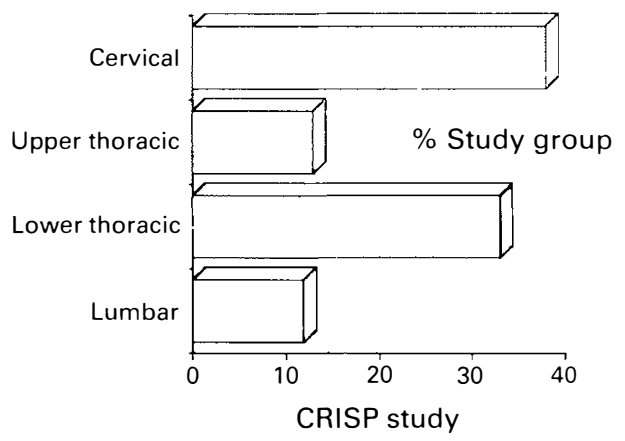

Figure 1 Level of spinal injury expressed as a percentage of spinal cord injury patients studied.

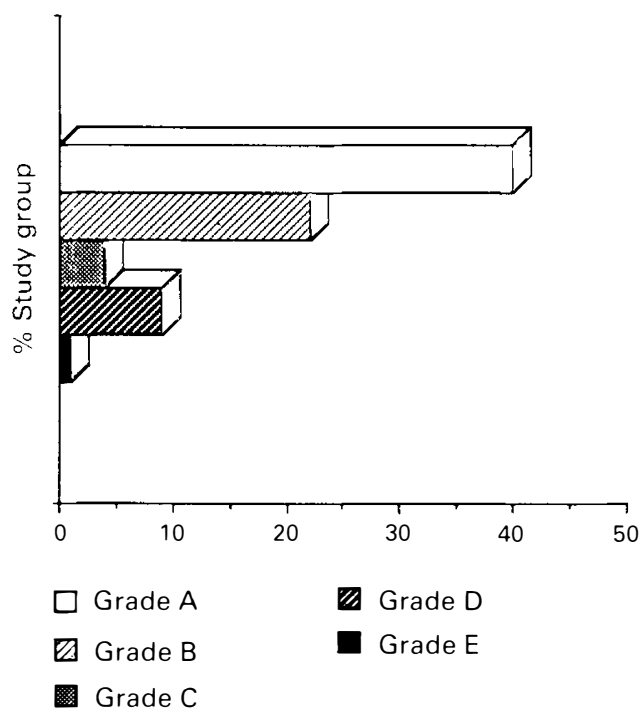

Figure 2 Frankel grade of completeness of spinal injury (Grade $\mathrm{A}=$ complete motor and sensory deficit to Grade $E=$ no deficit). Most study subjects had complete (Grade A) levels of injury.

on weight reduction, diabetic and low fat diets (Fig 4).

\section{Cardiac risk factor measurement}

Comparison of body weight in males according to age and all females is shown in Figure 5 . Spinal patients weighed slightly less overall but there was no significant difference between the two groups $(p=0.23$ male, $p=0.62$ female).

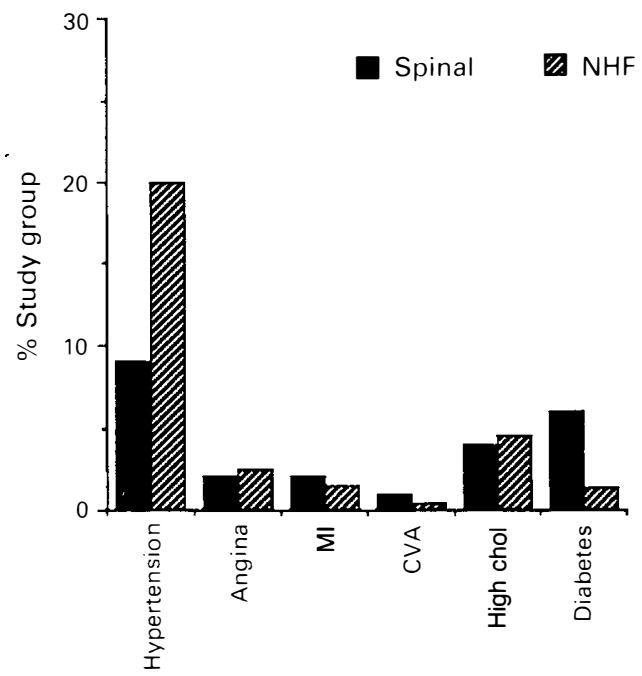

Figure 3 Percentage of spinal or National Heart Foundation study group subjects with known history of cardiovascular diseases: hypertension, angina, myocardial infarction (MI), cerebrovascular accident (CVA), hypercholesterolemia (High chol) or diabetes. There were fewer hypertensives but a greater percentage of diabetics in the spinal cord injury group.

There was a significant reduction in systolic blood pressure in male spinal patients compared to NHF controls (Fig 6) $(p=$ $0.015)$. Diastolic blood pressure was significantly lower in male and female spinal patients (Fig 7) $(p=0.011, p=0.016$ respectively). Similar levels were noted in spinal patients greater than 10 years post spinal injury for both systolic and diastolic blood pressure.

Total cholesterol levels were similar between the 2 groups (Fig 8) ( $p=0.25$ male, $p=0.09$ female) although slightly lower in the spinal group. There was no significant correlation between level of lesion and total cholesterol level (Fig 9) $(r=0.24, \quad p=$ 0.49).

HDL cholesterol was found to be significantly lower in male $(p=0.045)$ but not female $(p=0.075)$ spinal patients compared to NHF controls (Fig 10). HDL cholesterol levels were lower still in those patients greater than 10 years post spinal cord injury $(p=0.012$ ), (one way analysis of variance with pairwise comparison by 


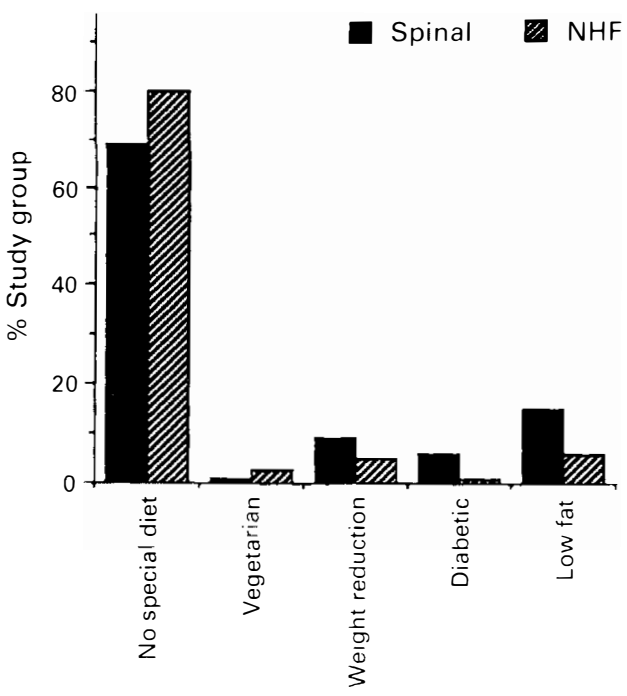

Figure 4 Percentage of spinal or National Heart Foundation study group subjects undertaking special diets ie vegetarian, weight reduction, diabetic, low fat or on no special diet. Both study groups were relatively well matched for dietary habits, although there was a somewhat higher percentage of spinal cord injury patients on diabetic and low fat diets.

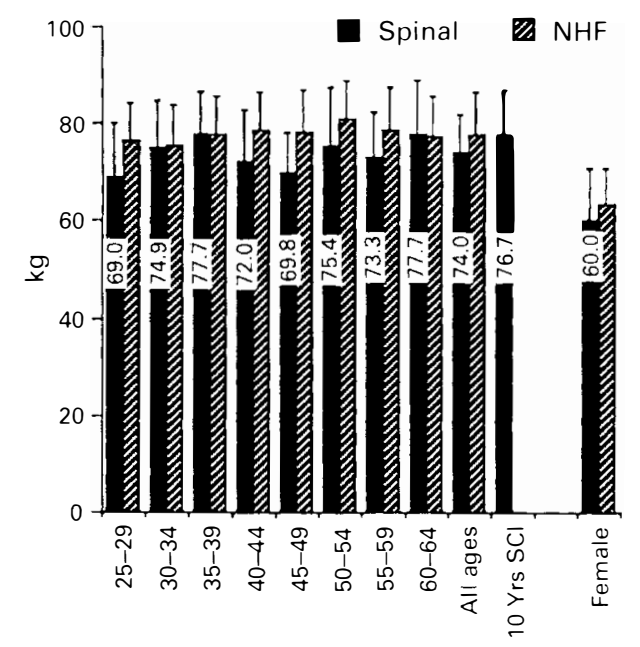

Figure 5 Body weight $(\mathrm{kg})$ of spinal and $\mathrm{Na}$ tional Heart Foundation study group subjects according to age in males, as well as for all males and females (mean \pm standard deviation). Mean values for the spinal group are displayed numerically. There were no significant differences between the 2 groups amongst both males and females (Student's unpaired t-test).

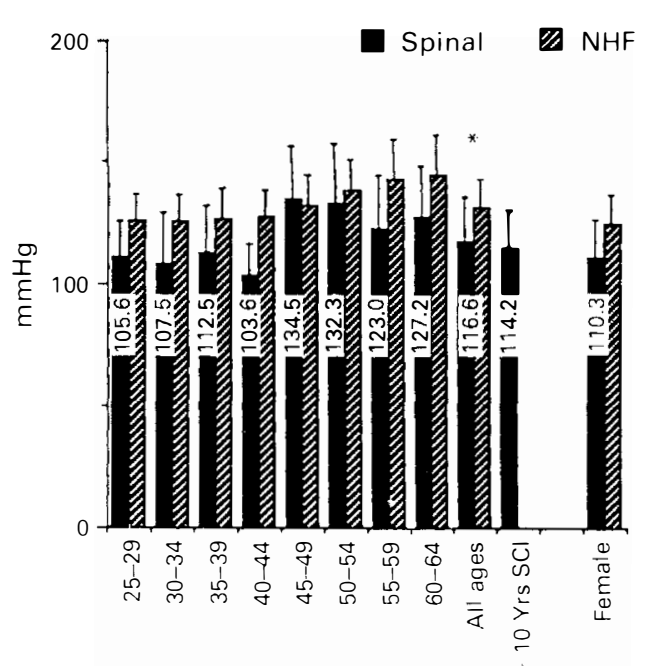

Figure 6 Systolic blood pressure $(\mathrm{mm} \mathrm{Hg})$ of spinal and National Heart Foundation study group subjects according to age in males, as well as for all males, subjects with spinal cord injury for greater than 10 years and all females (mean \pm standard deviation). Mean values for the spinal group are displayed numerically. Systolic blood pressure was significantly lower in males $(p<0.05)$ but not females in the spinal cord injury group (Student's unpaired t-test).

analysis of simple effects). No significant correlation was found between HDL cholesterol and level of lesion $(r=0.12, p=0.76)$ (Fig 11). However, when the HDL cholesterol level was related to the semi-quantitative assessment of level of exercise (Fig 11) a non significant trend was noted directly correlating exercise level and HDL cholesterol level $(r=0.54, p=0.086)$.

Similar levels of smoking were found between NHF controls, all spinal patients and spinal patients greater than 10 years post spinal injury for both male and female study subjects $(p=0.32, p=0.75$ respectively, Kruskal-Wallis nonparametric test, Fig 12). Plasma glucose levels, and independent risk factor for cardiovascular disease, was very similar between the 2 groups $(p=0.19$ male, $p=0.87$ female, Fig 13).

When the patients were assigned cardiovascular risk factor scores based on the MRFIT formula (Fig 14), male and female 


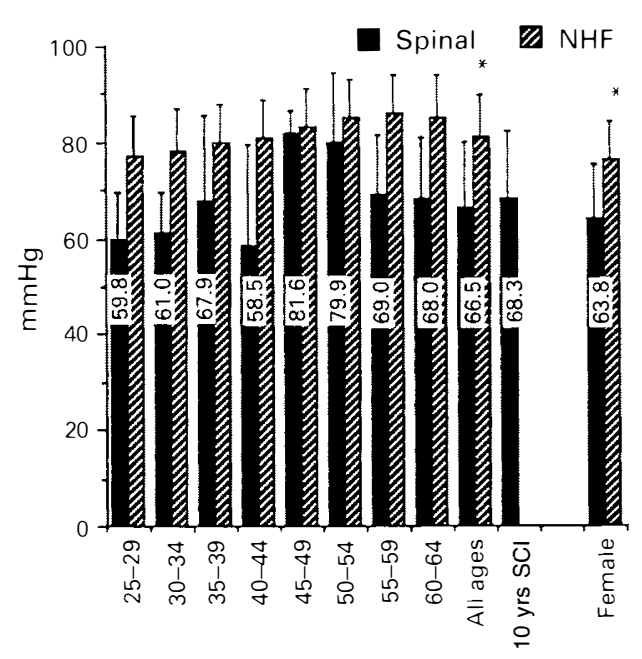

Figure 7 Diastolic blood pressure $(\mathrm{mmHg})$ of spinal and National Heart Foundation study group subjects according to age in males, as well as for all males, subjects with spinal cord injury for greater than 10 years and all females (mean \pm standard deviation). Mean values for the spinal group are displayed numerically. Diastolic blood pressure was significantly lower in males $(p<0.05)$ and females $(p<0.05)$ in the spinal cord injury group (Student's unpaired t-test).

spinal patients fell below the fiftieth percentile for all age groups.

\section{Discussion}

We have found low cardiac risk factor scores in spinal cord injury patients despite reports of increased cardiovascular disease incidence in these patients.

The major contribution to the low cardiac risk factor score in the spinal patients were low levels of diastolic blood pressure. While it would not seem likely that the mean diastolic blood pressure of 64 found in the SCI group would substantially reduce cardiovascular risk compared to the general normotensive population, a recent metaanalysis of epidemiological studies which examined the association between diastolic blood pressure and cardiovascular morbidity and mortality demonstrated a straight

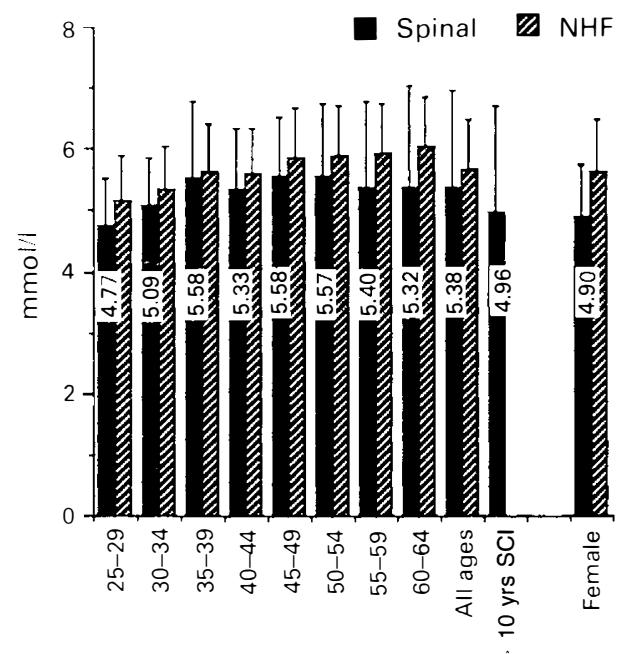

Figure 8 Total cholesterol levels $(\mathrm{mmol} / \mathrm{L})$ of spinal and National Heart Foundation study group subjects according to age in males, as well as for all males, subjects with spinal cord injury for greater than 10 years and all females (mean \pm standard deviation). Mean values for the spinal group are displayed numerically. There were no significant differences between the 2 groups amongst both males and females (Student's unpaired t-test).

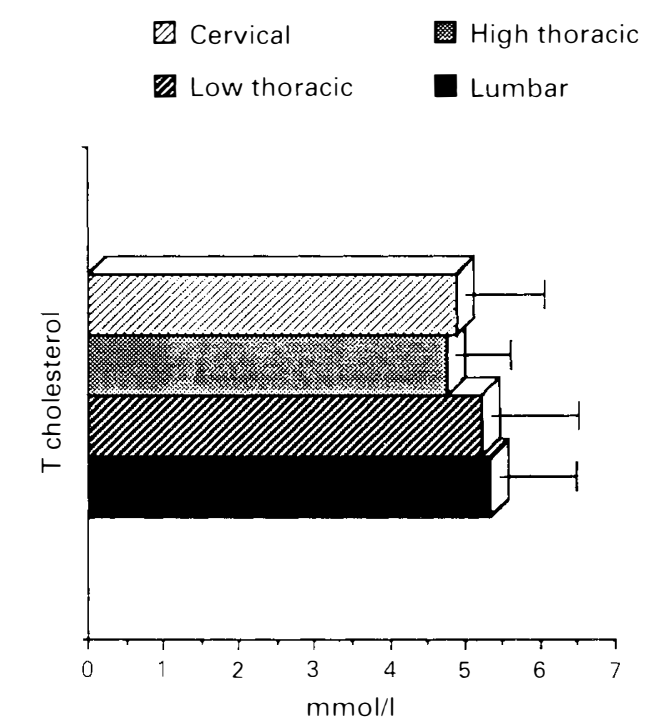

Figure 9 Total cholesterol levels $(\mathrm{mmol} / \mathrm{L})$ for all spinal cord injury patients according to level of spinal lesion (mean \pm standard deviation). There was no significant correlation between level of lesion and total cholesterol level. 


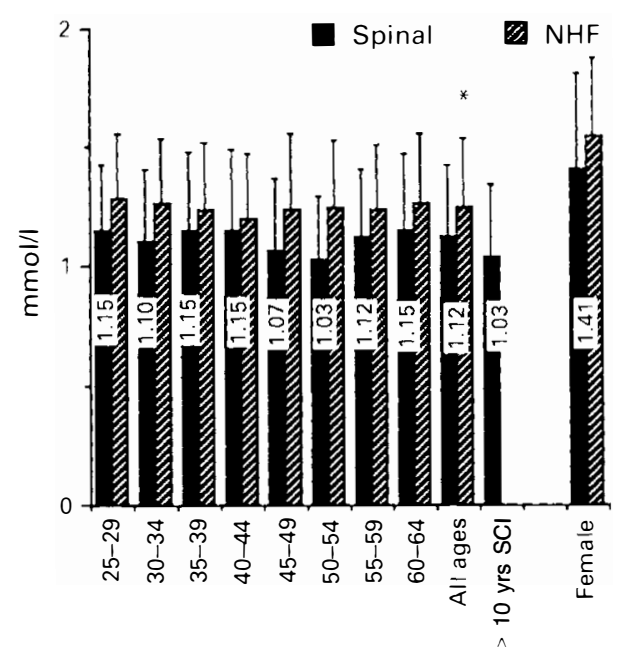

Figure 10 High density lipoprotein (HDL) cholesterol levels of spinal and National Heart Foundation study group subjects according to age in males, as well as for all males, subjects with spinal cord injury for greater than 10 years and all females. (mean \pm standard deviation). Mean values for the spinal group are displayed numerically. HDL cholesterol was significantly lower in males $(p<0.05)$ but not females in the spinal cord injury group in comparison to NHF study subjects. HDL cholesterol levels were lower still in those patients greater than 10 years post spinal injury $(p<0.05)$. (One way analysis of variance with pair-wise comparison by analysis of simple effects).

line relationship which included very low levels of diastolic blood pressure. ${ }^{10}$ There appears to be no J curve effect of increasing cardiac risk with very low levels of blood pressure.

As total cholesterol levels and levels of cigarette consumption were similar in these patients, the low risk is attributed entirely to their low diastolic blood pressures. It may be, however, that a more reliable assessment of cardiovascular risk would include pre rather than post spinal injury blood pressure levels.

The cardiac risk factor score derived in this way does not take into account other, increasingly recognised, risk factors for cardiovascular disease. The most important of these in the spinal population is that of HDL
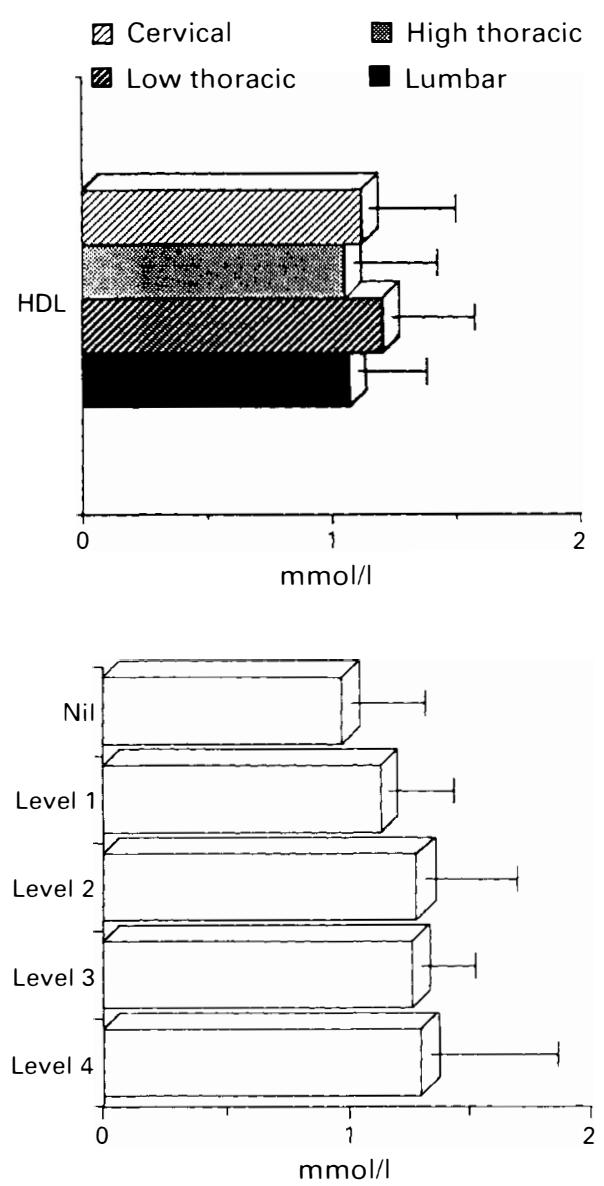

Figure 11 Upper panel: high density lipoprotein (HDL) cholesterol level for all spinal cord injury patients according to level of lesion. (mean \pm standard deviation). There was no significant correlation between level of lesion and HDL cholesterol level. Lower panel: high density lipoprotein (HDL) cholesterol level according to a semi-quantitative assessment of exercise level in spinal patients $($ NIL $=$ no exercise, level $4=$ wheelchair athlete). (Mean \pm standard deviation). There was a trend for a direct correlation between level of exercise and HDL cholesterol level but this correlation did not reach statistical significance.

cholesterol levels. Studies have found a direct correlation between level of physical activity and HDL level ${ }^{6}$ and our study has also found this association. As HDL is inversely related to cardiovascular disease, it is likely that the significantly lower levels 


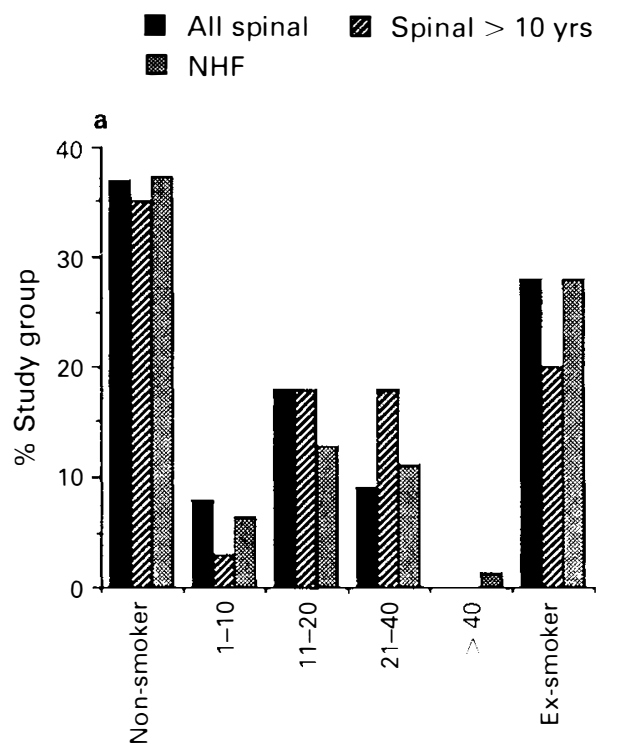

Spinal NHF

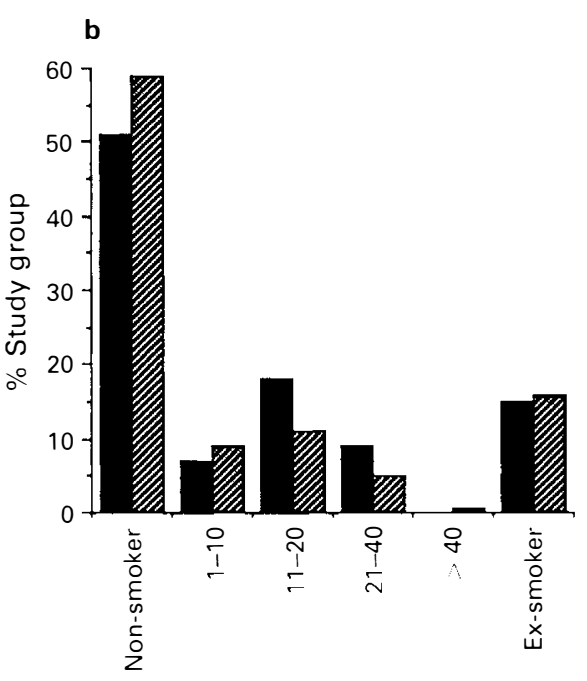

Figure 12 Current smoking status in all spinal patients, spinal patients greater than 10 years post injury and National Heart Foundation study subjects (upper panel $=$ males, lower panel $=$ females). Study subjects were grouped into ex-smokers, those who had never smoked and current smokers $(1-10,11-20,21-40$ or $>40$ cigarettes per day). Smoking status was similar in all 3 groups (Kruskal-Wallis nonparametric test).

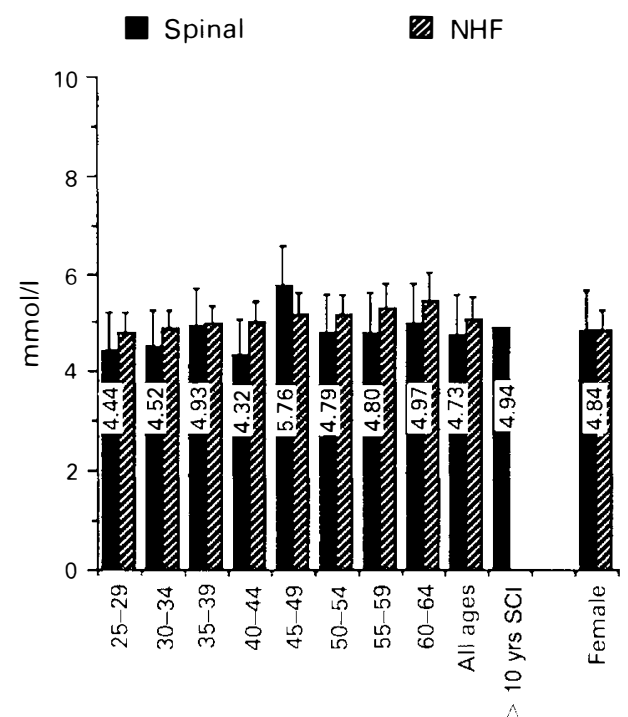

Figure 13 Fasting plasma glucose levels $(\mathrm{mmol} / \mathrm{L})$ of spinal or National Heart Foundation study group subjects according to age in males, as well as for all males, subjects with spinal cord injury for greater than 10 years and all females (mean \pm standard deviation). Mean values for the spinal group are displayed numerically. There were no significant differences between the 2 groups amongst both males and females (Student's unpaired t-test).

found in the spinal population may contribute to an increased cardiovascular disease risk.

We have documented increased blood pressure variability in spinal cord injury patients, particularly those with recent episodes of autonomic hyperreflexia. ${ }^{11}$ Increased blood pressure variability may be an additional risk factor for cardiovascular disease. Patients with increased blood pressure variability have been found to have increased end organ damage at the same mean level of blood pressure as those patients without increased blood pressure variability. ${ }^{12}$

Another explanation for the low cardiac risk factor score in our study may be related to the population studied. There may be fundamental differences between our Australian population and that of the Israeli population studied by Yekutial et al.$^{2}$ These 


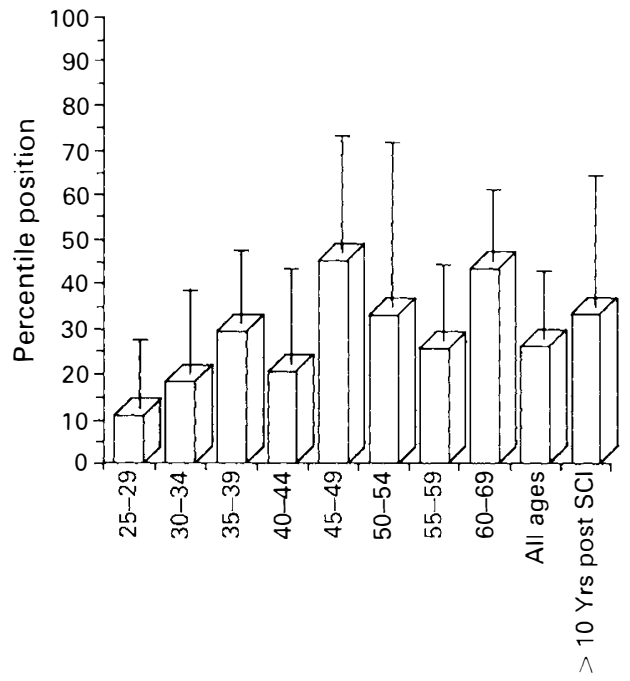

Figure 14 Cardiac Risk Factor Score in age specific percentiles in all spinal patients as well as those greater than 10 years post injury. Percentile position in comparison to age matched subjects from the National Heart Foundation risk factor prevalence study ${ }^{8}$ (mean \pm standard deviation). Spinal cord injury patients (including those greater than 10 years post injury) occupied a percentile position in the lower $50 \%$ of risk in all age groups. differences may relate to variations in diet as well as in lifestyles and culture.

It may also be that population attitudes to health, diet and exercise have changed in the last decade, placing the spinal population in a higher percentile position of cardiovasculr risk compared to the contempary general population.

\section{Conclusion}

We found no increase in levels of the well recognised cardiac risk factors of total cholesterol, cigarette consumption and blood pressure in spinal patients compared to an age and sex matched general population despite a reported increase incidence of cardiovascular disease in these patients. These findings documented an overall low cardiac risk factor score for spinal patients within the Australian population. This suggests that if SCI patients do indeed have an increased incidence of cardiovascular disease, it would appear to be due to factors other than those well recognised risk factors described.

\section{References}

1 National Heart Foundation of Australia (1986) Heart Facts Report: 23-27.

2 Yekutiel M, Brooks ME, Ohry A, Yarom J, Carel R (1989) The prevalence of hypertension, ischaemic heart disease and diabetes in traumatic spinal cord injured patients and amputees. Paraplegia 27: 58-62.

3 Geisler WO, Jousse AT, Wynne-Jones M. Breithaupt D (1983) Survival in traumatic spinal cord injury. Paralegia 21: 364-373.

4 Multiple Risk Factor Intervention Trial Research Group (1982) Multiple risk factor intervention trial: risk factor changes and mortality results. JAMA 248: 1465-1476.

5 Castelli WP (1984) Epidemiology of coronary heart disease: the Framingham study. Am J Med 76(2A): 4-12.

6 Laporte RE, Brenes G, Dearwater S, Murphy MA, Cauley JA, Dietrick R, Robertson R (1983) HDL cholesterol across a spectrum of physical activity from quadriplegia to marathon running. Lancet 1: 1212-1213.

7 Anderson KM, Castelli WP, Levy D. (1987) Cholesterol and mortality: 30 years of follow up from the Framingham study. JAMA 257: 2176-2180.

8 National Heart Foundation of Australia (1983) Risk Factor Prevalence Study Report No. 2.

9 McNeil JJ, Farish SJ, Simpson J (1988) Expressing Cardiovascular Risk as a Percentile Value. Department of Social and Preventive Medicine, Monash University.

10 McMahon S, Peto R, Cutler J, Collins R, Sorlie P, Neaton J et al (1990) Blood pressure, stroke, and coronary heart disease: part 1, prolonged differences in blood pressure: prospective observational studies corrected for the regression dilution bias. Lancet 335: 765-774.

11 Krum H, Howes LG, Brown DJ, Louis WJ (1989) Blood pressure variability in quadriplegic patients with autonomic hyperreflexia. Paraplegia 27: 284-288.

12 Mancia G, Parati G, Albini F, Villani A (1988) Circadian blood pressure variations and their impact on disease. J Cardiovasc Pharmacol 12(suppl 7): S11--S17. 